\title{
Status of the Test System of the MWPC for the LHCb Muon System
}

\author{
Massafferri A., Carboni G. and Santoveti E., Universita' di Roma - Tor Vergata, INFN
}

Nobrega R. and Bocci V., Universita' di Roma - La Sapienza, INFN

\begin{abstract}
This document describes the apparatus and the procedure used to test the 1368 Multi Wire Proportional Chambers (MWPC) after front-end assembly for the LHCb Muon System. Given a fully equipped chamber, this system is able to diagnose every channel through an analysis of front-end output drivers and noise rate versus threshold response. In addition, it verifies if the noise rate at the operational threshold is within appropriate limits. Aiming at an automatic, fast and userfriendly system for mass production tests of MWPC, the project has foreseen as well electronic identification of every chamber and front-end board, and data archiving in such a way to make it available to the Experiment Control System (ECS). The results obtained on about 130 chambers tested are described.
\end{abstract}

\section{INTRODUCTION}

The $\mathrm{LHCb}$ is an experiment dedicated to measure precisely the $\mathrm{CP}$ violation parameters and search for $\mathrm{B}$ mesons rare decays. An efficient muon detection is a fundamental requirement since many decays containing muons in the final state are sensitive to $\mathrm{CP}$ violation as well as to theories beyond Standard Model.

The LHCb Muon System [1]-[2] is divided in 5 stations (labelled as M1 up to M5), according to the distance to the interaction point, which one divided into 4 regions (R1 up to R4), according to the distance to the central axis. Except the M1R1 all chambers in LHCb Muon System are composed by two 2-gaps Multi Wire Proportional devices. Signals are collected from either anode (wire readout) or cathode (pad readout). The granularity (pad dimension) is based on the expected flux of the muon particles. The input capacitance as seen by the front-end electronics (FEE) depends of the chamber type and can vary from roughly $40 \mathrm{pF}$ to $250 \mathrm{pF}$.

The on-detector circuitry is composed by the SparkProtection board (SPB) and the front-end electronics called CARDIAC. The former board makes use of passive components while the latter processes and digitalizes chamber signals. The CARDIAC accommodates two dedicated 8channels Charge Amplifier-Shaper-Discriminator (ASD) radhard chip, named CARIOCA [3], and one chip, named DIALOG [4], developed for readout and control purposes. The CARIOCA has been developed to process both polarities, each one with slightly different signal responses. The DIALOG controls the 16 channels independently, using DAC to set thresholds, 24-bit internal counters and features for timing adjustments of signals.

\section{FRONT-END FEATURES}

For noise immunity purpose the CARIOCA discriminator makes use of a Differential Threshold Voltage (DTV) circuit. A Nominal Threshold, varying from 0 to $1.2 \mathrm{~V}$, provides two reference bias levels with opposite derivatives. The Effective Threshold is obtained from the absolute value of the subtraction of these two references values. It presents a minimum value of about $40 \pm 5 \mathrm{mV}$, called Resbias, which corresponds to approximately $810 \pm 10 \mathrm{mV}$ if expressed in the Nominal Threshold units, called Offset. The Fig. 1 shows the DTV features and the relation between the Nominal and Effective thresholds. Also represents the noise rate pattern during the threshold scan.

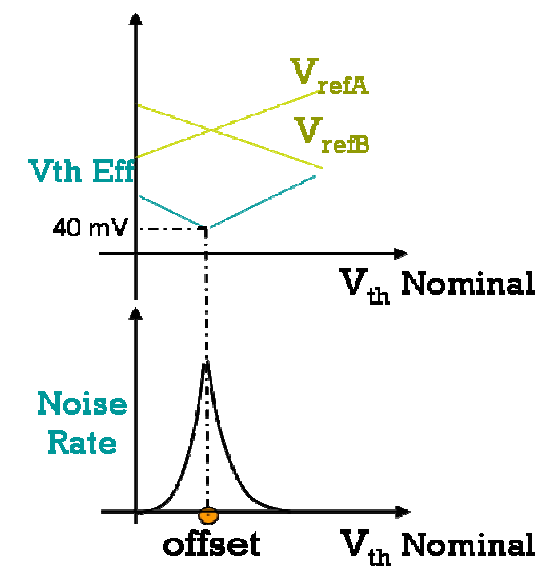

Fig. 1. Relation between the Effective Threshold and the Nominal Threshold, characteristic of the DTV discriminator of CARIOCA. It also shows the Noise Rate with respect to the Nominal Threshold. The Analysis of the threshold scan data is a fundamental tool of the Test procedure.

The CARIOCA band-width is been designed to operate with input capacitance up to $250 \mathrm{pF}$ (saturation region), which means a maximum rate of $25 \mathrm{MHz}$ for anode readout and 10 $\mathrm{MHz}$ for cathode readout. In addition the CARIOCA sensitivity and Equivalent-Noise-Charge (ENC) vary from 16 $\mathrm{mV} / \mathrm{fC}$ and $0.5 \mathrm{fC}$ at minimum input capacitance and $8 \mathrm{mV} / \mathrm{fC}$ and $2 \mathrm{fC}$ at maximum input capacitance respectively, as shown in Fig. 2. 

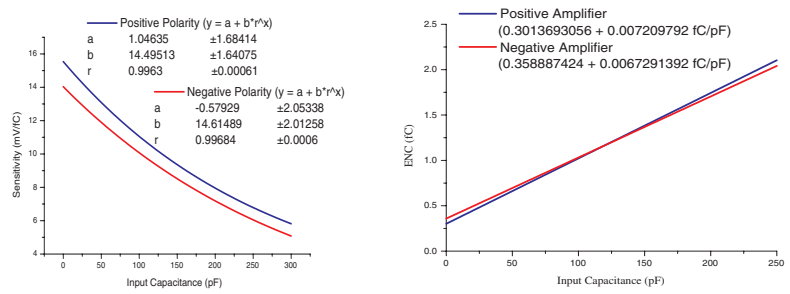

Fig.2. The sensitivity and Equivalent-Noise-Charge FEE parameters as a function of the input capacitance for the cathode (blue) and anode (red) readout.

\section{THE NOISE ANALYSIS}

One of the main parameters measured by the Test procedure is the chamber capacitance once is more sensitive to the typical problems during the assembling period, as shortcircuit, bad connectivity and others. In order to test the whole system comprised by chamber and front-end electronics, instead of a direct capacitance measurement, we have estimated the capacitance of each channel using its straight correlation with the noise signals readout in different threshold levels.

\section{A. Threshold Scan and Equivalent-Noise Estimation}

The probability density function describing the amplifier response in the presence of Gaussian noise of amplitude $V$ can be expressed by

$$
f_{n}(V)=\frac{1}{\sqrt{2 \pi} \sigma_{n}} \exp ^{-\frac{1}{2} \frac{V^{2}}{\sigma_{n}^{2}}}
$$

where $\sigma_{n}$ is the Equivalent-Noise in Volt units (ENV).

Therefore the factor in which the noise rate is reduced under the action of the threshold $V_{t h}$ is given by the error function

$$
\frac{f_{n}}{f_{n 0}}=\int_{V_{t h}}^{\infty} \frac{1}{\sqrt{2 \pi} \sigma_{n}} \exp ^{-\frac{1}{2} \frac{V^{2}}{\sigma_{n}^{2}}} d V
$$

The combined probability function for Gaussian time and amplitude distributions yields the expression for the noise rate as a function of threshold-to-ENV ratio as in (3).

$$
\frac{f_{n}}{f_{n 0}}=\exp ^{-V_{t h}^{2} / 2 \sigma_{n}^{2}}(3)
$$

As pointed out by Spieler [5] $f_{n 0}$ can be determined by the upper limit of the band-width $\omega_{\max }$, as expressed by Equation 4.

$$
f_{n 0} \cong \frac{\omega_{\max }}{\sqrt{3}}
$$

As a consequence the occupancy of noise hits, $\Phi$, in a time interval $\Delta t$ is given by

$$
\Phi=\frac{\omega_{\max } \Delta t}{\sqrt{3}} \exp ^{-\frac{V_{t h}^{2}}{2 \sigma_{n}^{2}}}
$$

It allows us to estimate ENV and the upper limit band-width using respectively the slope and the normalization parameters obtained from the fit of the noise collected at different threshold values modeled as a Gaussian function with the origin appropriately positioned at $V_{t h}=0$.

\section{B. The Capacitance Estimation}

The scan of the threshold permits a direct measurement of ENV. Firstly the Nominal threshold provided by DTV feature of CARIOCA must be converted in terms of Effective Threshold by the values found for Offset and Resbias.

The front-end input capacitance is then obtained from the ENV estimation in by solving a third degree equation given by the sensitivity (second degree) and the ENC (linear) capacitance dependence as shown in Figure 1. However this absolute measurement provides a quite large uncertainty. Therefore we defined a second type of capacitance, Exp Cap, measurement in which we use a value of the sensitivity parameter fixed on the expected capacitance value of the chamber. We observed that the bias inserted does not provide any mis-alarming in diagnostic tool.

This analysis has been implemented and has been utilized as a diagnostic tool for $\mathrm{LHCb}$ Muon Chamber Readout Electronics.

\section{Test SetuP}

The test setup consists of a MWPC full equipped with front-end boards, a barcode reader, a control unit device and readout circuitry, all supervised by a PC.

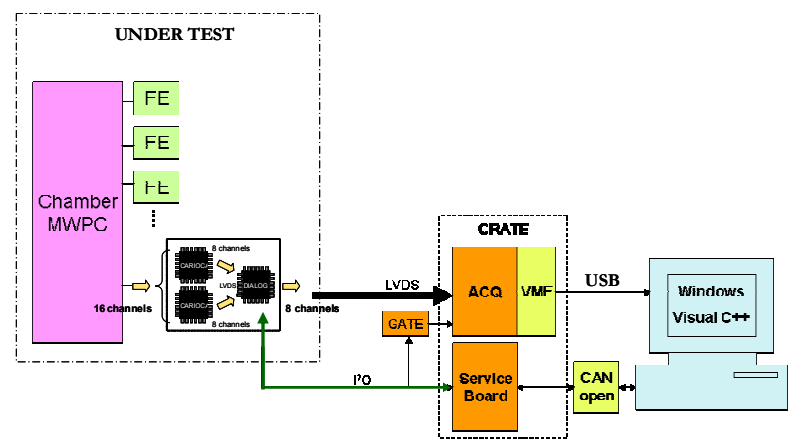

Fig. 3. The schematic view of the setup required by the Test procedure.

\section{A. FEE Control system}

The control of the front-end is performed through a Service Board [6]. It can access in parallel all FEE of a given chamber, generating pulses to be used mainly to inject signals and to control internal and external counters. This board is accessed using the CANopen protocol, specifically a commercial adapter manufactured by Kvaser Company.

\section{B. Readout Electronics}

The FEE signals can be readout internally, through the scaler feature presented in DIALOG, or externally using the 64-channels counting module ACQ [7] with a CAEN VME v1718 interface. A gate-board has been designed and used in order to translate the LVDS signal, sent by the Service Board module to the DIALOG as time gate, into a TTL signal, compatible with ACQ logic. 


\section{Barcode Reader}

Before to enclose the chamber within the Faraday Cage, a barcode reader and ROOT (CERN analysis software) based program is used to identify and link the chamber with the set of front-end used during assembling.

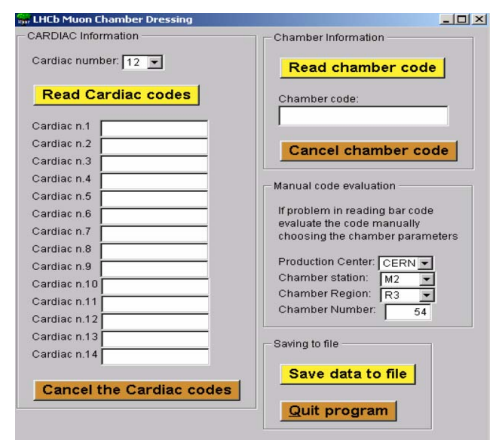

Fig. 4. The ROOT graphic format of the program responsible for the creation of the barcode files.

\section{Software}

A Visual C++-ROOT integrated software tool has been developed to control all circuits, performing the diagnostic analysis of chambers and visualization of results.

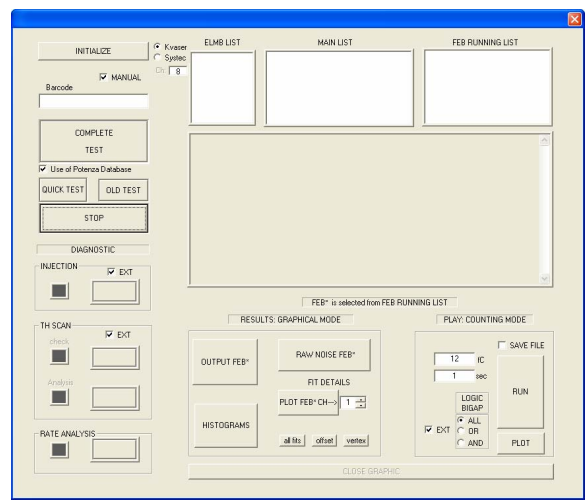

Fig. 5. The main frame of the Visual $\mathrm{C}++$ software for the Test procedure. We can see, at left, the sequence of steps of the procedure, each one with its respective led and buttons to show the diagnostic messages. At the center and botton, we can see the graphical interface that present the variety of parameters available as an output of the Test. A right and botton, we can see the facility in which we perform the acquisition using any threshold value and during any gate time. It is also possible set the logical between 2-gaps.

\section{Procedures And Diagnostic}

The Test procedure is ready to be run after the creation of the barcode file of the chamber. By clicking the initialization and "complete test" button the user process the FEE recognition and initiate with the chain of Tests described below. After about 5 minutes the Test finishes and the diagnostic is available in monitor as well as properly archived.

\section{A. Cable checking and Pulse Injection}

After setting the threshold of all channels to the maximum, 100 pulses are injected in a first channel of each FEE in order to check if they are been externally readout by the correct FEE. After checking if the cable configuration are properly done, a simultaneous injection of 500 pulses is performed in each channel of the chamber. The counting rates given by the DIALOG and the ACQ external scaler are compared. The scope of this step is able to verify a huge sort of problems as the output LVDS linea of the FEE, cable installation (swap), false contacts, bad ground quality, cross-talk. This step has shown to be very useful also in the pit.

\section{B. Threshold Scan}

The noise acquisition, during a gate time of $200 \mathrm{~ms}$, of all threshold values, in steps of $2.35 \mathrm{mV}$, is performed for all channels of the chamber.

\section{1) Noise Presence and Open Channels}

A first analysis is carried out checking if the number of measured points obtained during the threshold scan is sufficient in order to fit the data. This is a useful tool to single out dead channels and open channels. If no points at all are found, the channel is marked as dead. If the maximum noise point, defined as offset, is found but with an unsatisfactory number of threshold points around it, the channel is marked as Open Channel, a problem likely related to bad connectivity.

\section{2) Offset pattern recognition}

From the threshold scan, a sequence of offset values can be obtained for the 16 channels of each FEE. This pattern is compared with the results obtained in the front-end mass production test, in order to check if the FEE on the chamber has been correctly identified. The pattern information can be also used to identify chambers during their assemblage on the experimental area.
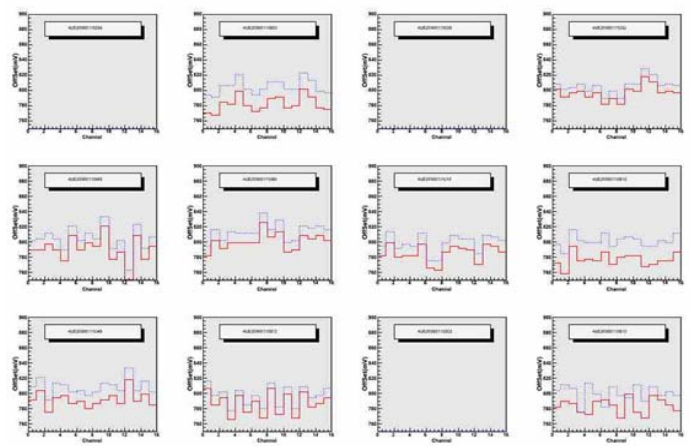

Fig. 6. The Offset pattern of 12 FEE. Each histogram contains the 16 Offset values obtained by threshold scan during the Test procedure and equivalent ones obtained previously in stand-alone mass production tests of the front-end electronics. Note that the first, the third and the eleventh FEE barcode was not found in the FEE database.

\section{3) Noise Rate versus Threshold}

After a fit of experimental noise data with respect to the Effective Threshold (requiring offset and Resbias parameters), modeled using (5), the Equivalent-Noise-Volt is calculated. Using the calibration curve (Figure 4) this value is converted directly into an estimate of detector capacitance. Then, the calculated capacitance values for all channels are averaged and the mean value should be within 3 standard deviations from the nominal capacitance for the specific type of chamber under test.

The diagnostic routine subsequently verifies if the capacitance of every channel is comprised inside 3 standard 
deviations with respect to the mean value measured. If one or more channels are out of range, an alarm is raised and the abnormal values are printed specifying their source.

\section{Noise rate at the nominal threshold}

The LHCb Muon TDR [1] requires a maximum noise rate of $100 \mathrm{~Hz}$ per channel for MWPC. Recent studies of $\mathrm{LHCb}$ Muon Detector have shown that channels with a noise rate up to a few $\mathrm{kHz}$ are not critical. In addition, a muon efficiency of $99 \%$ is observed by using a threshold at $8 \mathrm{fC}$ for cathode readout and $14 \mathrm{fC}$ for anode readout. The diagnostic tool measures the electronics noise rate at those threshold values during 1 second in order to check if is within the $1 \mathrm{KHz}$ limit. Beside the error alarm the diagnostic also warn the user in the case of the noise rate is outside the limit for threshold of $7 \mathrm{fC}$ (cathode readout) and $12 \mathrm{fC}$ (anode readout).

\section{RESULTS}

Up to now 100 M5R4 (220 pF), 22 M5R3 (140 pF) as well as 6 M5R2 $(120 \mathrm{pF})$ chambers has been tested using the automatic Test System in the LNF (Frascati National Laboratory). More than $10 \%$ of them required some sort of intervention to fix problems, as dead channels, bad connections, short-circuit, defects in SPB, bad I2C control cables. All of them have been properly spotted and diagnosed by the Test procedure. The capacitance measurements as well as the noise rate at threshold equal to 8 and $14 \mathrm{fC}$, respectively for cathode and anode readout, are shown in Fig. A slightly shift of the capacitance mean value with respect to the simulated value is expected given the complexity of the system under study. The spread on the capacitance measurement is mainly due to variation into input FEE parameters.
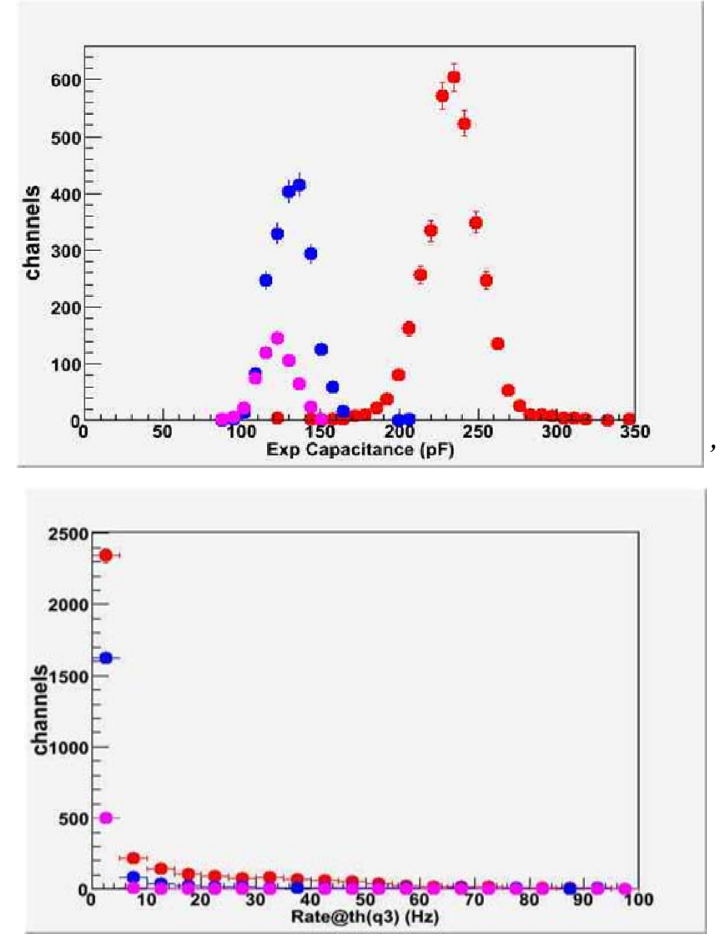

Fig. 7. Results of the Test procedure of the 100 M5R4 (red), 22 M5R3 (blue) and 6 M5R2 (pink) chambers performed at LNF. On the top we can see the capacitance measurement while, on the botton, the noise rate at operational threshold.

In order to verify the reliability of the Test procedure based on the noise analysis we performed a cross-check using charged particles from Cosmic. In order to take off the electronic noise a coincidence of hits between two 2-gaps is required. The acquisition is done for different values of HighVoltage in order to spot the plateau of high efficiency, desired signature of well behavior of MWPC.

From 15 M5R4 chambers studied in this way we observed that $0.5 \%$ of the channels presented low muon efficiency as shown by Fig, while two chambers do not presented a satisfactory plateau region (both with at least one case of low efficiency channel). After prompt studied of these pathologic chambers we detected the presence of short-circuits which leaves a clear signature at the top of the rate distribution. The Test Procedure failed to detect these defective channels due the utilization of a maximum rate cut of $2 \mathrm{MHz}$. A re-analysis of all available data using the upgraded version of Test procedure, in which this cut was removed, was able to detect all those problems.

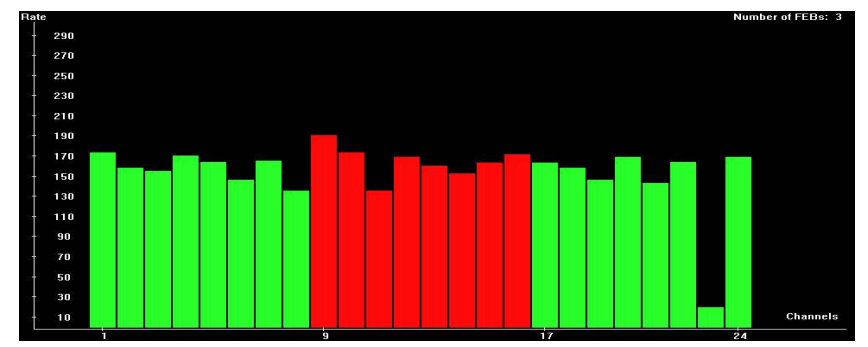

Fig. 8. Cosmic counting rate at operational threshold, HV and gas flux, of three FEE of a given M5R4 chamber. The 8 output LVDS readouts of each front-end electronics define the $\mathrm{x}$ axis and correspond to the counting rate of two 2-gaps in $25 \mathrm{~ns}$ coincidence. We can easily observe a low efficiency channel in the last FEE.

\section{CONCLUSION}

A fast (about 5 minutes) and automatic Test procedure has been successfully developed for the test of the more than 1300 MWPC of the LHCb Muon System.

The procedure has shown to be very effective to spot problems in the assemblage of the 128 chambers tested up to now, providing an important guide to solve them. A cosmic acquisition has also been used to check the reliability of the method and point out that $0.5 \%$ of the approved channels present a muon efficiency out of the specifications. A retest using the Upgrade Version of the Test System has been able to discriminate all those cases.

Four Test System Units has been installed, two at CERN, one at LNF and one on the experimental area in order to minimize drastically the installation of problematic chambers. 


\section{REFERENCES}

[1] LHCb Collaboration, "LHCb Muon System TDR", CERN/LHCC 2001010, 2001.

[2] LHCb Collaboration, "Addendum to the Muon System TDR", CERN/LHCC 2003-002, 2003.

[3] N. Pelloux, "Architecture \& Layout Overview", June 2003, unpublished.

[4] S. Cadeddu, et al, Nuclear Instruments and Methods in Physics Research $A$, pp 486, 2004.

[5] H. Spieler, "Rate of Noise Hits in Threshold Discriminator Systems", Lecture Notes, Physics 198, Spring Semester UC Berkeley, 1998.

[6] V. Bocci, et al, "Service Board - Datasheet", INFN sezione Roma, Italy, 2003, unpublished.

[7] A. Balla, et al, "ACQ Manual”, INFN LNF, Italy, 2003, unpublished. 